Diabetologe $2021 \cdot 17: 677$

https://doi.org/10.1007/s11428-021-00801-4

Online publiziert: 16. August 2021

๑) Springer Medizin Verlag $\mathrm{GmbH}$, ein Teil von

Springer Nature 2021
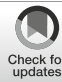

\section{Erratum zu: Diabetes Update 21. Neue Erkenntnisse auf dem Gebiet des Diabetes mellitus}

\author{
Werner Scherbaum
}

Universitätsklinikum, Heinrich-Heine-Universität Düsseldorf, Düsseldorf, Deutschland

\section{Erratum zu:}

Diabetologe 2021

https://doi.org/10.1007/s11428-021-

00782-4

Der Untertitel und die erste Überschrift des Beitrags waren fehlerhaft angegeben worden.

Bitte beachten Sie den korrekten Beitragstitel Diabetes Update 21. Neue Erkenntnisse auf dem Gebiet des Diabetes mellitus (Diabetes Update 21. Advancements in the area of diabetes mellitus) und die korrekte erste Überschrift des Beitrags Pathophysiologie und Prävention des Typ-2-Diabetes.

Die Redaktion bittet, den Fehler zu entschuldigen und um Beachtung der korrekten Version.

Der vollständige und korrigierte Artikel steht Ihnen auf www.springermedizin.de zur Verfügung. Bitte geben Sie dort den Beitragstitel in die Suche ein.

\section{Korrespondenzadresse}

Univ.-Prof. Dr. med. Werner Scherbaum Universitätsklinikum, Heinrich-HeineUniversität Düsseldorf

Moorenstraße 5, 40225 Düsseldorf,

Deutschland

scherbaum@uni-duesseldorf.de
Die Online-Version des Originalartikels ist unter https://doi.org/10.1007/s11428-021-00782-4 zu finden.

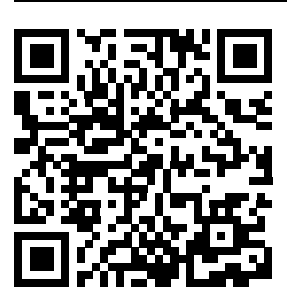

QR-Code scannen \& Beitrag online lesen 